\title{
Subject Monitoring
}

National Cancer Institute

\section{Source}

National Cancer Institute. Subject Monitoring. NCI Thesaurus. Code C156639.

The act of observing, recording, and reporting the status, safety, and performance of clinical trial participants as specified in the study protocol. 